\section{Factores asociados a una baja calidad de vida en adultos chilenos durante la cuarentena por COVID-19}

\author{
EDUARDO GUZMÁN-MUÑOZ ${ }^{1, \mathrm{a}, \mathrm{d}}$, YENY CONCHA-CISTERNAS $^{1,2, \mathrm{a}, \mathrm{e}}$, \\ ARIEL OÑATE-BARAHONA ${ }^{3, a, e}$, CARLOS LIRA-CEA $^{1, \mathrm{~b}}$, \\ IGOR CIGARROA-CUEVAS ${ }^{1, a, d}$, GUILLERMO MÉNDEZ-REBOLLEDO $^{1, a, d}$, \\ MARCELO CASTILLO-RETAMAL ${ }^{4, \mathrm{c}, \mathrm{d}}$, PABLO VALDÉS-BADILLA ${ }^{2, \mathrm{c}, \mathrm{d}}$, \\ RAFAEL ZAPATA-LAMANA ${ }^{5, \mathrm{c}, \mathrm{d}}$
}

\section{Factors associated with low quality of life in Chilean adults during the COVID-19 quarantine}

Background: The quarantine and social distancing implemented during COVID 19 pandemic may hamper the quality of life of the population. Aim: To determine the factors associated with a low quality of life during COVID 19 quarantine in Chilean adults. Material and Methods: The SF-36 survey about quality of life was answered by 1,082 Chilean adults aged between 18 and 60 years, who were quarantined by the COVID-19 health alert. Other variables studied were sociodemographic background, nutritional status, lifestyles, level of physical activity, sedentary behavior and sleep hours. Risk factors associated with low quality of life were identified by logistic regression analysis for each of the 8 dimensions evaluated in the SF-36 questionnaire. Results: The factors that increased the probability of having a lower general health perception were being female $($ Odds ratio $(O R)=1.29 ; p=0.05)$, being physically inactive $(O R=2.76$ $p<0.01)$, unhealthy hours of sleep $(O R=1.58, p<0.01)$, smoking $(O R=1.59$, $p<0.01)$ and eating junk food $(O R=2.26 ; p<0.01)$. For the other dimensions of quality of life, the most frequently repeated factors were being female, junk food consumption, and being physically inactive and sedentary. Conclusions: There are factors associated with a low quality of life during the quarantine of the COVID-19 pandemic in the Chilean population. Their identification could reinforce remedial actions at the government level to benefit the health of the population during this health emergency.

(Rev Med Chile 2020; 148: 1759-1766)

Key words: Life Style; Quality of Life; Quarantine; SARS-CoV-2.

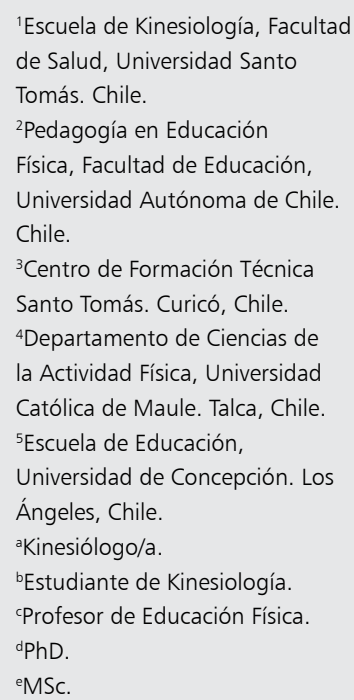

Los autores declaran no tener conflictos de interés.

Trabajo no recibió financiamiento.

Recibido el 30 de junio de 2020, aceptado el 10 de noviembre de 2020.

Correspondencia a: Eduardo Guzmán Muñoz eguzmanm@santotomas.cl
$\mathrm{E}$ n diciembre del año 2019 hubo un brote de una enfermedad respiratoria de etiología desconocida reportada por primera vez en Wuhan, China ${ }^{1}$. La Organización Mundial de la Salud (OMS) identificó un nuevo coronavirus denominado SARS-CoV-2 como el virus causante de la pandemia que se propagó rápidamente en todo el mundo ${ }^{1}$. En Latinoamérica, países como Brasil, Perú y Chile fueron quienes más casos positivos de COVID-19 han presentado entre marzo y mayo del $2020^{2}$.

Debido a la pandemia, las autoridades sanitarias han implementado diversas medidas para evitar la propagación del virus, entre ellas 
destacan la cuarentena y el distanciamiento social, las cuales buscan limitar el contacto e interacción entre las personas ${ }^{3,4}$. Sin embargo, según lo observado en otras pandemias (SARS 2002 y MERS 2012), estas medidas preventivas podrían ocasionar trastornos en aspectos mentales y emocionales de las personas $s^{5,6}$. Actualmente, en China se ha reportado una disminución de la percepción de la calidad de vida y salud mental en personas adultas que han sido sometidas a cuarentena preventivas por COVID-19, siendo el pánico y la ansiedad generada por la pandemia las principales causas de los trastornos observados en la población estudiada ${ }^{7}$.

La calidad de vida corresponde a la percepción de bienestar que tiene la persona respecto a su salud física, psicológica y social, y a las expectativas que tiene en relación a ella ${ }^{8}$. Para evaluar la percepción de la calidad de vida relacionada con la salud en personas adultas, uno de los instrumentos más utilizados es el cuestionario SF-36 (36-item Medical Outcomes Study Short-Form General Health Survey) ${ }^{9}$. Este cuestionario ha sido utilizado recientemente para evaluar personas que padecen COVID-19 en países orientales ${ }^{10}$, no obstante, en países occidentales, información de la percepción que tienen los individuos con respecto a su salud durante la actual pandemia es limitada. Se ha propuesto que la calidad de vida puede estar influenciada por los hábitos y estilos de vida de las personas ${ }^{11}$, comportamientos que podrían alterarse negativamente debido a la cuarentena por la pandemia del COVID-19 ${ }^{12}$. En consideración a todo lo expuesto, el objetivo de este estudio fue determinar los factores asociados a una baja calidad de vida durante la cuarentena por la pandemia del COVID-19 en la población adulta chilena.

\section{Materiales y Métodos}

\section{Diseño y participantes de estudio}

Estudio descriptivo de corte transversal, desarrollado en el mes de mayo del 2020. Este contó con la aprobación del comité de ética de la Universidad Santo Tomás, Chile (código 45-20).

La población estuvo compuesta por habitantes chilenos con edades comprendidas entre los $18 \mathrm{y}$ 60 años, que estuvieran en cuarentena preventiva por alerta sanitaria de COVID-19 (voluntaria o impuesta por las autoridades sanitarias), y que no se encontraran con diagnóstico o sospecha de COVID-19. Los participantes fueron invitados a participar a través de afiches divulgados en redes sociales, los cuales contaban con un enlace a una plataforma online (Google forms) que contenía los cuestionarios utilizados en esta investigación (disponible entre el 30 de mayo y 6 de junio de 2020). Todos los participantes aprobaron un consentimiento informado previo a la recolección de los datos en esta misma plataforma. El tipo de muestreo fue no probabilístico casual, pues la selección de los participantes respondió a la facilidad y voluntad de acceso de los participantes. De 1.335 personas que aceptaron participar, 1.082 cumplieron con los criterios de inclusión y exclusión, conformando la muestra de esta investigación.

\section{Variables}

Las variables estudiadas fueron: antecedentes sociodemográficos, estado nutricional, estilos de vida, nivel de actividad física, conducta sedentaria, horas de sueño y percepción de la calidad de vida. Los antecedentes sociodemográficos consideraron: edad, género y procedencia geográfica (rural o urbana). El peso corporal y la estatura bípeda fueron autoreportadas. Investigaciones previas han demostrado que el autoreporte de la estatura y peso es considerado un método aceptable y satisfactorio para su uso en estudios de vigilancia epidemiológica ${ }^{13,14}$. El índice de masa corporal (IMC) fue calculado dividiendo el peso corporal por la estatura bípeda al cuadrado $\left(\mathrm{kg} / \mathrm{m}^{2}\right)$. A partir del IMC, la variable estado nutricional se dicotomizó en normopeso $\leq 24,9 \mathrm{~kg} / \mathrm{m}^{2}$ y exceso de peso $\geq 25,0 \mathrm{~kg} / \mathrm{m}^{2}{ }^{15}$. Para los datos asociados a estilos de vida, como consumo de alcohol, comida chatarra y hábito tabáquico, se utilizó la clasificación señalada en la Encuesta Nacional de Salud 2009-2010 15 , para después categorizar a los participantes en los dominios "consume" y "no consume".

El nivel de actividad física se midió a través del Cuestionario Internacional de Actividad Física (IPAQ) versión corta ${ }^{16}$. El indicador de actividad física total fue expresado de manera continua en METs (Metabolic-energy-equivalents) y se dicotomizaron los resultados en físicamente inactivos cuando el gasto energético fue $\leq 599 \mathrm{METs} / \mathrm{min} /$ semana y físicamente activos cuando el gasto energético fue $\geq 600 \mathrm{METs} / \mathrm{min} / \mathrm{semana}^{17,18}$. Se consi- 
deró conducta sedentaria cuando los participantes destinaban un tiempo $\geq 4 \mathrm{~h}$ diarias al desarrollo de actividades sentados, como ha sido señalado en otros estudios en población chilena ${ }^{19,20}$.

Las horas de sueño fueron auto-reportadas a través de la siguiente pregunta: "En promedio, ¿Cuántas horas duermes por día?”21. La variable continua se dicotomizó en "horas de sueño saludable" cuando las respuestas indicaban 7 y 8 horas de sueño promedio al día y en "horas de sueño no saludables" cuando la respuesta se encontraba fuera de aquel rango ${ }^{22,23}$.

Para evaluar la percepción de la calidad de vida se utilizó el cuestionario de salud SF-369. El SF-36 es un instrumento de autoreporte que contiene 36 preguntas de 8 dimensiones relacionadas con la salud de las personas: función física, rol físico, dolor corporal, vitalidad, función social, rol emocional, salud mental y salud general ${ }^{9}$. El puntaje obtenido corresponde a valores en una escala de 0 a 100. Un mayor puntaje refleja una mejor calidad de vida9 . Para categorizar estos valores se calculó la media aritmética de los participantes en cada una de las 8 dimensiones de la calidad de vida evaluadas, y a partir de esta medida, los datos fueron dicotomizados en "bajo la media" y "sobre la media". Este procedimiento de categorización ha sido utilizado en estudios previos de calidad de vida ${ }^{24,25}$.

\section{Análisis de datos}

Los datos fueron analizados con el software estadístico SPSS 25.0 (SPSS 25.0 para Windows, SPSS Inc., IL, USA). Los datos de caracterización de la población estudiada son presentados como promedio y desviación estándar para variables continuas, y como porcentaje para variables categóricas. Los factores de riesgo asociados a una baja calidad de vida se identificaron mediante un análisis de regresión logística para cada una de las 8 dimensiones evaluadas en el cuestionario SF-36. Estos análisis fueron ajustados por estado nutricional, edad y procedencia geográfica, y presentados como Odds Ratio con sus respectivos intervalos de confianza de 95\% (95\% CI). Se verificó la colinealidad de las variables presentes en los análisis mediante valores menores a 0,10 de tolerancia y valores sobre 10,0 de factor de inflación de la varianza (FIV) para confirmar no existencia de multicolinealidad. El nivel de significancia fue definido como $\mathrm{p}<0,05$.

\section{Resultados}

Las características generales de los participantes de este estudio se exhiben en la Tabla 1, mientras que las medias de los puntajes obtenidos en las dimensiones de percepción de la calidad de vida se muestran en la Tabla 2.

Los análisis de regresión logística para las dimensiones función física, rol físico, dolor corporal y vitalidad de la calidad de vida se resumen en la Figura 1. El género femenino se asoció a una mayor probabilidad de presentar una disminución de la calidad de vida en las dimensiones función física $(\mathrm{OR}=1,40 ; \mathrm{p}=0,025)$ y dolor corporal $(\mathrm{OR}=1,35 ; \mathrm{p}=0,018)$. Por su parte, la inactividad

Tabla 1. Características generales de la muestra

\begin{tabular}{|lc|}
\hline & n $=\mathbf{1 . 0 8 2}$ \\
\hline Edad (media y DE) & $29,2 \pm 8,1$ \\
\hline Género femenino (\%) & 65,3 \\
\hline Género masculino (\%) & 34,7 \\
\hline Procedencia urbana (\%) & 83,5 \\
\hline Exceso de peso (\%) & 45,9 \\
\hline Físicamente inactivo/a (\%) & 32,8 \\
\hline Sedentario/a (\%) & 75,6 \\
\hline Consumo de tabaco (\%) & 18,5 \\
\hline Consumo de alcohol (\%) & 51,8 \\
\hline Consumo de comida chatarra (\%) & 15,1 \\
\hline Horas de sueño entre 7 y 8 (\%) & 29,3 \\
\hline
\end{tabular}

DE: desviación estándar.

Tabla 2. Resultados de las dimensiones de percepción de la calidad de vida $(n=1.082)$

\begin{tabular}{|lcccc|}
\hline & Media & DE & $\begin{array}{r}\text { \% sobre } \\
\text { la media }\end{array}$ & $\begin{array}{c}\text { \% bajo } \\
\text { la media }\end{array}$ \\
\hline Función física & 93,5 & 12,8 & 74,6 & 25,4 \\
\hline Rol físico & 88,3 & 25,5 & 76,4 & 23,6 \\
\hline Dolor corporal & 74,7 & 24,1 & 52,7 & 47,3 \\
\hline Vitalidad & 56,8 & 19,1 & 47,9 & 52,1 \\
\hline Función social & 42,5 & 14,7 & 55,3 & 44,7 \\
\hline Rol emocional & 50,4 & 44,1 & 48,7 & 51,3 \\
\hline Salud mental & 61,9 & 19,4 & 50,3 & 49,7 \\
\hline Salud general & 53,6 & 15,2 & 56,9 & 43,1 \\
\hline
\end{tabular}

DE: desviación estándar. 
física se asoció a una mayor probabilidad de presentar una baja calidad de vida en las dimensiones función física $(\mathrm{OR}=2,49 ; \mathrm{p}=0,001)$, rol físico $(\mathrm{OR}=1,52 ; \mathrm{p}=0,005)$, dolor corporal $(\mathrm{OR}=2,22$; $\mathrm{p}=0,001)$ y vitalidad $(\mathrm{OR}=3,22 ; \mathrm{p}=0,001)$.
El consumo de comida chatarra y la conducta sedentaria también fueron factores asociados a una disminución de la calidad de vida en estas dimensiones durante la cuarentena preventiva por COVID-19 (Figura 1).

\begin{tabular}{|c|c|c|c|c|}
\hline \multicolumn{5}{|l|}{ a) Función física } \\
\hline Género femenino- & $\sum_{-1}$ & 1.40 & $1.04-1.89$ & 0.025 \\
\hline Físicamente inactivo/a- & $\mapsto-1$ & 2.49 & $1.86-3.31$ & 0.001 \\
\hline Sedentario/a- & 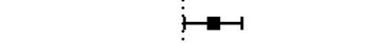 & 1.44 & $1.02-2.02$ & 0.038 \\
\hline Consumo de tabaco- & $\stackrel{\vdots}{\vdots}$ & 1.15 & $0.81-1.63$ & 0.419 \\
\hline Consumo de alcohol- & $\mapsto$ & 0.91 & $0.63-1.36$ & 0.322 \\
\hline Consumo comida chatarra- & $\longmapsto$ & 2.31 & $1.49-3.59$ & 0.001 \\
\hline 7-8 horas de sueño- & 1 & 0.86 & $0.64-1.17$ & 0.354 \\
\hline 0.1 & $\underset{\text { Baja probabilidad }}{\stackrel{\text { Alta probabilidad }}{\longrightarrow}}$ & 10 & & \\
\hline \multicolumn{5}{|c|}{ Odds ratio } \\
\hline \multicolumn{5}{|l|}{ b) Rol físico } \\
\hline Género femenino- & 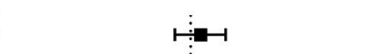 & 1.13 & $0.83-1.52$ & 0.418 \\
\hline Físicamente inactivo/a- & $\mapsto-1$ & 1.52 & $1.13-2.04$ & 0.005 \\
\hline Sedentario/a & $\mapsto$ & 0.82 & $0.59-1.14$ & 0.243 \\
\hline Consumo de tabaco- & $\mapsto-1$ & 0.90 & $0.62-1.31$ & 0.603 \\
\hline Consumo de alcohol- & $\stackrel{\vdots}{\mapsto-1}$ & 1.02 & $0.70-1.39$ & 0.122 \\
\hline Consumo comida chatarra- & $\stackrel{\vdots}{\longrightarrow}$ & 1.47 & $0.93-2.33$ & 0.095 \\
\hline $7-8$ horas de sueño- & $\mapsto$ & 1.08 & $0.79-1.48$ & 0.618 \\
\hline 0.1 & $1 \underset{\text { Baja probabilidad }}{\stackrel{\text { Alta probabilidad }}{\longrightarrow}}$ & 10 & & \\
\hline \multicolumn{5}{|c|}{ Odds ratio } \\
\hline \multicolumn{5}{|l|}{ c) Dolor corporal } \\
\hline Género femenino- & 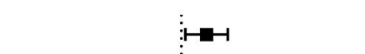 & 1.35 & $1.05-1.74$ & 0.018 \\
\hline Físicamente inactivo/a- & $\mapsto-1$ & 2.22 & $1.71-2.88$ & 0.001 \\
\hline Sedentario/a & $\stackrel{\vdots}{\leftrightarrow}=-1$ & 1.29 & $0.97-1.71$ & 0.078 \\
\hline Consumo de tabaco- & $\mapsto$ & 1.09 & $0.80-1.49$ & 0.554 \\
\hline Consumo de alcohol- & $\stackrel{\vdots}{-}$ & 1.06 & $0.79-1.49$ & 0.302 \\
\hline Consumo comida chatarra- & $\vdots \longmapsto$ & 1.82 & $1.19-2.80$ & 0.006 \\
\hline $7-8$ horas de sueño- & ing & 1.10 & $0.94-1.29$ & 0.215 \\
\hline 0.1 & $\underset{\text { Baja probabilidad }}{\longleftarrow} \underset{\text { Alta probabilidad }}{\longrightarrow}$ & 10 & & \\
\hline \multicolumn{5}{|c|}{ Odds ratio } \\
\hline d) Vitalidad & & OR & IC $95 \%$ & p \\
\hline Género femenino- & $\stackrel{\vdots}{\vdots}$ & 1.22 & $0.95-1.57$ & 0.111 \\
\hline Físicamente inactivo/a- & $\mapsto-1$ & 3.22 & 2.45-4.24 & 0.001 \\
\hline Sedentario/a- & $\mapsto-1$ & 1.90 & $1.43-2.53$ & 0.001 \\
\hline Consumo de tabaco- & $\stackrel{-10-1}{1}$ & 1.16 & $0.85-1.58$ & 0.343 \\
\hline Consumo de alcohol- & 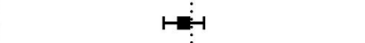 & 0.91 & $0.72-1.16$ & 0.490 \\
\hline Consumo comida chatarra & $\vdots \longmapsto$ & 2.17 & $1.38-3.41$ & 0.001 \\
\hline 7-8 horas de sueño- & 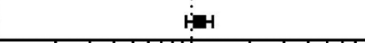 & 1.14 & $0.97-1.34$ & 0.091 \\
\hline & $\underset{\text { Baja probabilidad }}{\stackrel{\text { Alta probabilidad }}{\longrightarrow}}$ & 10 & & \\
\hline & Odds ratio & & & \\
\hline
\end{tabular}

Figura 1. Factores asociados a calidad de vida relacionada con la salud: a) función física, b) rol físico, c) dolor corporal y d) vitalidad. Datos presentados como odds ratio (OR) y sus respectivos $95 \%$ IC. El análisis fue ajustado por estado nutricional, edad y procedencia geográfica. Un OR $>1$ indica que hay una mayor probabilidad de tener una baja calidad de vida. Se consideró significativo un valor $\mathrm{p}<0,05$. 
Los análisis de regresión logística para las dimensiones función social, rol emocional, salud mental y salud general de la calidad de vida se resumen en la Figura 2. El género femenino se asoció a una mayor probabilidad de presentar una disminución de la calidad de vida en la dimensión salud general $(\mathrm{OR}=1,29 ; \mathrm{p}=0,046)$. Para la variable inactividad física, existió una mayor probabilidad de presentar una baja calidad de vida en las dimensiones función social $(\mathrm{OR}=1,31$;

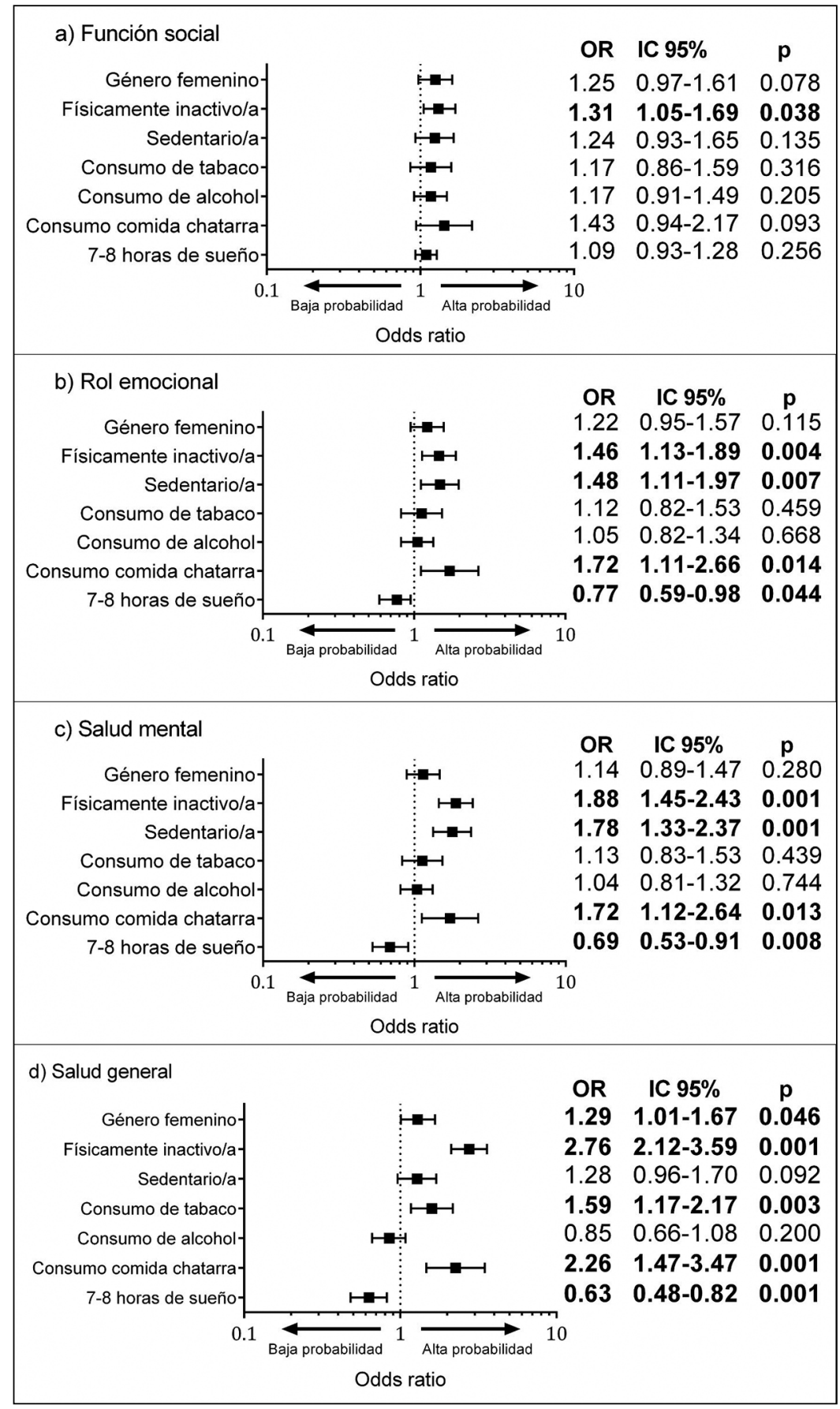

Figura 2. Factores asociados a calidad de vida relacionada con la salud: a) función social, b) rol emocional, c) salud mental y d) salud general. Datos presentados como odds ratio (OR) y sus respectivos $95 \%$ IC. El análisis fue ajustado por estado nutricional, edad y procedencia geográfica. Un OR > 1 indica que hay una mayor probabilidad de tener una baja calidad de vida. Se consideró significativo un valor $\mathrm{p}<0,05$. 
$\mathrm{p}=0,038)$, rol emocional $(\mathrm{OR}=1,46 ; \mathrm{p}=0,004)$, salud mental $(\mathrm{OR}=1,88 ; \mathrm{p}=0,001)$ y salud general $(\mathrm{OR}=2,76 \mathrm{p}=0,001)$. Con respecto a la conducta sedentaria, se pudo observar que existe una mayor probabilidad de presentar una baja calidad de vida en las dimensiones rol emocional $(\mathrm{OR}=1,48 ; \mathrm{p}=0,007)$ y salud mental $(\mathrm{OR}=1,78$; $\mathrm{p}=0,001)$. El consumo de tabaco, consumo de comida chatarra y horas de sueño también fueron factores asociados a una disminución de la calidad de vida en estas dimensiones durante la cuarentena preventiva por COVID-19 (Figura 2).

\section{Discusión}

Los principales resultados de este estudio señalan que existen factores asociados a género y estilos de vida que aumentan el riesgo de presentar una baja calidad de vida durante un periodo de cuarentena (voluntaria o impuesta por las autoridades) por la pandemia del COVID-19 en población adulta chilena. Respecto a la salud general, los factores que aumentan la probabilidad de tener una menor calidad de vida son ser de género femenino, ser físicamente inactivo, horas de sueño no saludables, consumo de tabaco y comida chatarra. Estudios previos han reportado que en situaciones de aislamiento o cuarentena por epidemias como SARS, MERS y COVID-19, las personas desarrollaron trastornos psicológicos asociados al estado de alarma, preocupación y sensación de miedo que provoca una pandemia, lo que repercute directamente sobre su percepción de calidad de vida ${ }^{5,6,10}$.

En nuestro estudio las mujeres presentaron un mayor riesgo de tener una baja calidad de vida en las dimensiones función física, dolor corporal y salud general. Similares resultados se han reportado en estudios anteriores, donde se ha considerado al género femenino como un factor de riesgo que aumenta la probabilidad de tener una menor calidad de vida durante la pandemia por COVID-19 ${ }^{10,26}$. En general, durante una pandemia ocurren cambios en la dinámica diaria familiar, en donde muchas mujeres deben asumir nuevos roles, como el cuidado total de los hijos (por no poder asistir a la escuela), que sumado a las labores domésticas generan una carga de estrés que atenta sobre su calidad de vida ${ }^{10}$. Del mismo modo, se ha informado que luego de eventos adversos y/o trau- máticos, como el aislamiento por una pandemia, los pensamientos negativos y recuerdos intrusivos son más frecuentes en mujeres que en hombres ${ }^{27,28}$.

Un factor que resultó ser influyente en todas las dimensiones de la calidad de vida evaluadas en este estudio fue el nivel de actividad física, sugiriendo que ser físicamente inactivo/a aumenta la probabilidad de tener una menor calidad de vida durante el período de cuarentena en una pandemia. Estos resultados coinciden con los entregados por Nguyen y cols. quienes reportaron una asociación positiva entre realizar actividad física con la calidad de vida relacionada con la salud en personas que se encuentran en cuarentena por COVID-19 ${ }^{10}$. Se ha demostrado que la práctica de actividad física promueve un mejor autoconcepto físico y niveles de satisfacción con la vida, lo que repercute sobre el estado de animo de las personas y su percepción de la calidad de vida ${ }^{29,30}$. Estos efectos se explican debido a la acción estimulante de la actividad física sobre el sistema de opioides endógenos lo que induce un incremento de la concentración de ß-endorfinas y consecuente sensación de bienestar y disminución de la ansiedad-estrés ${ }^{30,31}$. Asimismo, la práctica regular de actividad física se relaciona con mejor manejo de enfermedades crónicas no transmisibles, lo que induce un mayor estado de satisfacción con la salud general del individuo, y por lo tanto, una mejor percepción de la calidad de vida relacionada con la salud ${ }^{32}$.

Otros resultados de esta investigación señalan que conductas no saludables como el consumo de tabaco, consumo de comida chatarra y horas de sueño no saludables también aumentan el riesgo de presentar una baja calidad de vida durante la cuarentena por COVID-19. De estas, el consumo de comida chatarra parece ser uno de los más influyentes, siendo un factor de riesgo en 6 de las 8 dimensiones de la calidad de vida evaluadas. Otros autores también han reportado que hábitos no saludables como el consumo de comida chatarra y tabaco son factores de riesgo para la calidad de vida y salud de las personas en cuarentena por COVID-19 ${ }^{10,12}$. Mientras que una alimentación saludable se asocia con una menor probabilidad de depresión y deterioro de la calidad de vida ${ }^{33,34}$. Se ha planteado que una adecuada alimentación no solo mejora los parámetros nutricionales, sino también los aspectos funcionales ya que aporta energía y vitalidad ${ }^{35}$, lo que podría explicar la mejor calidad de vida autopercibida por las per- 
sonas de este estudio. Por otra parte, las horas de sueño no saludables (dormir menos de 7 horas o más de 8 horas) aumentan la probabilidad de tener baja calidad de vida, asociándose a un mayor estrés y ansiedad ${ }^{26}$. Por lo tanto, estos hábitos no saludables podrían exacerbar la percepción de los distintos aspectos que componen la salud como se observó en nuestros resultados.

Dentro de las limitaciones de esta investigación se encuentran la selección no probabilística causal de los participantes y la recolección de los datos mediante auto-reporte, lo cual podría restringir la validez externa de los resultados y ocasionar un sesgo por la infra o sobreestimación de los participantes en relación con las variables estudiadas, respectivamente.

\section{Conclusión}

Existen factores asociados a una menor calidad de vida durante la cuarentena por la pandemia del COVID-19 en la población chilena evaluada en este estudio. Los factores que reducen la percepción de calidad de vida en cuarentana son el género femenino, ser físicamente inactivo/a, presentar conducta sedentaria, horas de sueño no-saludables y consumo de comida chatarra. Desde una perspectiva de salud pública, es necesario reportar los factores que impactan la calidad de vida de las personas con la intención de reforzar acciones remediales a nivel gubernamental para beneficiar la salud de la población durante la pandemia.

\section{Referencias}

1. Wilder-Smith A, Chiew CJ, Lee VJ. Can we contain the COVID-19 outbreak with the same measures as for SARS?. Lancet Infect Dis 2020; 20 (5): E102-E7. DOI: https://doi.org/10.1016/S1473-3099(20)30129-8.

2. Undurraga EA, Chowell G, Mizumoto K. Case fatality risk by age from COVID-19 in a high testing setting in Latin America: Chile, March-May, 2020. medRxiv 2020. Doi: https://doi.org/10.1101/2020.05.25.20112904.

3. Wilder-Smith A, Freedman D. Isolation, quarantine, social distancing and community containment: pivotal role for old-style public health measures in the novel coronavirus (2019-nCoV) outbreak. J travel med 2020; 27 (2): taaa020. DOI: 10.1093/jtm/taaa020.

4. Parmet WE, Sinha MS. Covid-19-The Law and Limits of Quarantine. New Engl J Med 2020; 382 (15): e28. doi: 10.1056/NEJMp2004211.

5. Jeong H, Yim HW, Song YJ, Ki M, Min JA, Cho J, et al. Mental health status of people isolated due to Middle East Respiratory Syndrome. Epidemiol Health 2016; 38: 7. doi: 10.4178/epih.e2016048.

6. Reynolds DL, Garay JR, Deamond SL, Moran MK, Gold W, Styrai R. Understanding, compliance and psychological impact of the SARS quarantine experience. Epidemiol Infect 2008; 136 (7): 997-1007. doi: 10.1017/ S0950268807009156.

7. Zhang YF, Ma ZF. Impact of the COVID-19 Pandemic on Mental Health and Quality of Life among Local Residents in Liaoning Province, China: A Cross-Sectional Study. Int J Environ Res Public Health 2020; 17 (7): 2381. doi: 10.3390/ijerph17072381.

8. Urzua M A. Health related quality of life: Conceptual elements. Rev Med Chil 2010; 138 (3): 358-65. Doi: http://dx.doi.org/10.4067/S003498872010000300017.

9. Alonso J, Prieto L, Anto JM. The spanish version of the sf-36 health survey - a measure of clinical outcomes. Med Clin 1995; 104 (20): 771-6.

10. Nguyen HC, Nguyen MH, Do BN, Tran CQ, Nguyen TTP, Pham KM, et al. People with Suspected COVID-19 Symptoms Were More Likely Depressed and Had Lower Health-Related Quality of Life: The Potential Benefit of Health Literacy. J Clin Med 2020; 9 (4): 18. doi: 10.3390/ jcm9040965.

11. Agrawal R, D'Silva C. Assessment of quality of life in normal individuals using the SF36 questionnaire. Int J Cur Res Rev 2017; 9: 43-7.

12. Mattioli AV, Sciomer S, Cocchi C, Maffei S, Gallina S. Quarantine during COVID-19 outbreak: changes in Diet and physical activity increase the risk of cardiovascular disease. Nutr Metab Cardiovasc Dis 2020. Doi: https://doi.org/10.1016/j.numecd.2020.05.020.

13. Dekkers JC, van Wier MF, Hendriksen IJM, Twisk JWR, van Mechelen W. Accuracy of self-reported body weight, height and waist circumference in a Dutch overweight working population. Bmc Med Res Methodol 2008; 8: 69. doi: 10.1186/1471-2288-8-69.

14. Yoong SL, Carey ML, D'Este C, Sanson-Fisher RW. Agreement between self-reported and measured weight and height collected in general practice patients: a prospective study. Bmc Med Res Methodol 2013; 13: 38. doi: 10.1186/1471-2288-13-38.

15. OMS/OPS. Panorama de la Seguridad Alimentaria y Nutricional en América Latina y el Caribe. Organización de las Naciones Unidas para la Alimentación y la Agricultura, Organización Panamericana de la Salud. 
Santiago, Chile 2016. http://www.fao.org/3/a-i6747s.pdf.

16. Vinas BR, Barba LR, Ngo J, Majem LS. Validity of the international physical activity questionnaire in the Catalan population (Spain). Gac Sanit 2013; 27 (3): 254-7. doi: 10.1016/j.gaceta.2012.05.013.

17. Seron P, Munoz S, Lanas F. Levels of physical activity in an urban population from Temuco, Chile. Rev Med Chile 2010; 138 (10): 1232-9.

18. Ainsworth BE, Haskell WL, Herrmann SD, Meckes N, Bassett DR, Jr., Tudor-Locke C, et al. Compendium of Physical Activities: A Second Update of Codes and MET Values. Med Sci Sports Exerc 2011; 43 (8): 1575-81. doi: 10.1249/MSS.0b013e31821ece12.

19. Martínez MA, Leiva AM, Petermann F, Garrido A, Díaz $\mathrm{X}$, Álvarez C, et al. Correlates of sedentary behaviors in Chile: evidence from the National Health Survey 20092010. Rev Med Chile 2018; 146 (1): 22-31. doi: 10.4067/ s0034-98872018000100022.

20. Concha-Cisternas Y, Guzmán-Munoz E, Valdes-Badilla P, Lira-Cea C, Petermann F, Celis-Morales C. Levels of physical activity and excess body weight in university students. Rev Med Chile 2018; 146 (8): 840-9. doi: 10.4067/s0034-98872018000800840.

21. Martínez-Moya M, Navarrete-Munoz EM, de la Hera MG, Giménez-Monzo D, González-Palacios S, Valera-Gran D, et al. Association between hours of television watched, physical activity, sleep and excess weight among young adults. Gac Sanit 2014; 28 (3): 203-8. doi: 10.1016/j.gaceta.2013.12.003.

22. Wild CJ, Nichols ES, Battista ME, Stojanoski B, Owen AM. Dissociable effects of self-reported daily sleep duration on high-level cognitive abilities. Sleep 2018; 41 (12): 182. doi: 10.1093/sleep/zsy182.

23. Gottlieb DJ, Ellenbogen JM, Bianchi MT, Czeisler CA. Sleep deficiency and motor vehicle crash risk in the general population: a prospective cohort study. Bmc Med 2018; 16 (1): 44. doi: 10.1186/s12916-018-1025-7.

24. Tajvar M, Arab M, Montazeri A. Determinants of health-related quality of life in elderly in Tehran, Iran. Bmc Public Health 2008; 8: 323. doi: 10.1186/1471-2458-8323.

25. Yilmaz-Oner S, Oner C, Dogukan FM, Moses TF, Demir K, Tekayev N, et al. Anxiety and depression predict quality of life in Turkish patients with systemic lupus erythematosus. Clin Exp Rheumatol 2015; 33 (3): 360-5.

26. Altena E, Baglioni C, Espie CA, Ellis J, Gavriloff D,
Holzinger B, et al. Dealing with sleep problems during home confinement due to the COVID-19 outbreak: Practical recommendations from a task force of the European CBT-I Academy. J Sleep Res. 2020; e13052. DOI: 10.1111/jsr.13052.

27. McLean CP, Anderson ER. Brave men and timid women? A review of the gender differences in fear and anxiety. Clin psychol rev 2009; 29 (6): 496-505. doi: 10.1016/j.cpr.2009.05.003.

28. Soni M, Curran VH, Kamboj SK. Identification of a narrow post-ovulatory window of vulnerability to distressing involuntary memories in healthy women. Neurobiol Learn Mem 2013; 104: 32-8. doi: 10.1016/j. nlm.2013.04.003.

29. Concha-Cisternas Y, Castillo-Retamal M, Guzmán-Muñoz E. Comparación de la calidad de vida en estudiantes universitarios según nivel de actividad física. Univ Salud. 2020; 22 (1): 33-40. Doi: http://dx.doi.org/10.22267/ rus.202201.172.

30. Arruza JA, Arribas S, Gil DML, Irazusta S, Romero S, Cecchini JA. The impact of duration in sport and physical activity on the psychological well-being. Rev Int Med Cien Act Fis Deporte 2008; 8 (30): 171-83.

31. Bonet J, Parrado E, Capdevila L. Acute effects of exercise on mood and hrv. Rev Int Med Cien Act Fis Deporte 2017; 17 (65): 85-100. DOI: http://dx.doi.org/10.15366/ rimcafd2017.65.006.

32. Celis-Morales C, Salas C, Álvarez C, Aguilar Farias N, Ramírez Campillos R, Leppe J, et al. Higher physical activity levels are associated with lower prevalence of cardiovascular risk factors in Chile. Rev Med Chil 2015; 143 (11): 1435-43. http://dx.doi.org/10.4067/S003498872015001100009.

33. Molendijk M, Molero P, Sánchez-Pedreno FO, Van der Does W, Martínez-González MA. Diet quality and depression risk: A systematic review and dose-response meta-analysis of prospective studies. J Affect Disord 2018; 226: 346-54. doi: 10.1016/j.jad.2017.09.022.

34. Donlucas GM, Favela JAA, Morales MER, Esquivel JAV. Principales Factores de riesgo cardiovascular en escolares. Culcyt 2016; 54 (11): 72-80.

35. Rajmil L, López-Aguilá S, Mompart-Penina A. Calidad de vida relacionada con la salud y factores asociados al sobrepeso y la obesidad en la población infantil de Cataluña. Med Clin 2011; 137: 37-41. https://doi. org/10.1016/S0025-7753(11)70027-6. 